\title{
Left Sided Inferior Vena Cava in a Patient Diagnosed with Renal Cell Carcinoma
}

\author{
Charalampos Fragkoulis, loannis Glykas*, Dimitrios Moschotzopoulos, Aggeliki Leventi, \\ Georgios Papadopoulos, Georgios Stathouros and Konstantinos Ntoumas
}

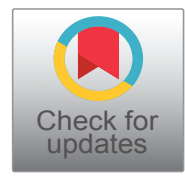

Urology Department, General Hospital of Athens "G. Gennimatas", Greece

*Corresponding author: loannis Glykas, MD, Department of Urology, General Hospital of Athens G. Gennimatas, Athens, Greece, Tel: +3069-8490-0729

\begin{abstract}
Inferior vena cava (IVC) is a major abdominal vein responsible for the transportation of deoxygenated blood from the lower extremities and the abdomen through diaphragm to the superior vena cava and eventually to the right atrium. IVC is located to the right of the midline and is an important anatomic structure in cases of operations involving the right kidney. In this paper we present a rare case of left sided IVC in a patient diagnosed with renal cell cancer who underwent open left partial nephrectomy.
\end{abstract}

\section{Introduction}

IVC is a large retroperitoneal vein responsible for the deoxygenated blood transportation from the lower extremities and the abdomen through diaphragm to the right atrium. Anatomically is formed by the confluence of the right and left iliac veins at the level of $L 5$ vertebrae. After its formation it lies along the right anterolateral aspect of the vertebral column and passes through the central tendon of the diaphragm around the T8 vertebral level [1]. IVC is an important anatomical structure and injuries may occur during operations of the right kidney such as nephrectomy or partial nephrectomy. Thus, urologists should be able to identify IVC preoperatively during imaging as well as intraoperatively in case of damage.

\section{Case Description}

We present a case of a 75 -year-old male who was referred to our department due to a $5 \mathrm{~cm}$ left renal tumor located in the lower pole. In the CT performed we recognized IVC located in the left of the midline as well as total aplasia of superior vena cava (Figure 1). Vein drainage above the diaphragm was performed through the vein system of azygos and hemiazygos veins. Left renal vein was drained straight to the IVC but as IVC was located to the left side, it did not pass in front of the aorta as usual (Figure 2). A successful open partial nephrectomy was performed by a retroperitoneal approach.

\section{Discussion}

Many anatomic anomalies of the IVC are described. Most clinically significant are left sided IVC, double IVC, intrahepatic IVC agenesis and total absence of infrarenal IVC [2]. Left sided IVC has a suspected prevalence up to $0.5 \%$ and it is caused by the regression of the right supracardinal vein and the persistence of the left supracardinal vein [3]. Although major vascular injuries involving IVC during laparoscopic or open partial nephrectomy are rare, it is of outmost importance to recognize such major anatomic landmarks and to identify anatomic anomalies prior to surgery in order to be able to face any intraoperative complication [4].

\section{Conclusion}

Left sided inferior vena cava is an exceedingly rare entity. Recognizing this rare anatomic variation before surgery through the imaging techniques is very important for the surgeon's preparation of possible intraoperative complications.

\section{Institutional Review Board}

The study was approved by the Ethics Committee of

Citation: Fragkoulis C, Glykas I, Moschotzopoulos D, Leventi A, Papadopoulos G, et al. (2020) Left Sided Inferior Vena Cava in a Patient Diagnosed with Renal Cell Carcinoma. Int J Radiol Imaging Technol 6:069. doi.org/10.23937/2572-3235.1510069

Accepted: November 11, 2020; Published: November 13, 2020

Copyright: (C) 2020 Fragkoulis C, et al. This is an open-access article distributed under the terms of the Creative Commons Attribution License, which permits unrestricted use, distribution, and reproduction in any medium, provided the original author and source are credited 


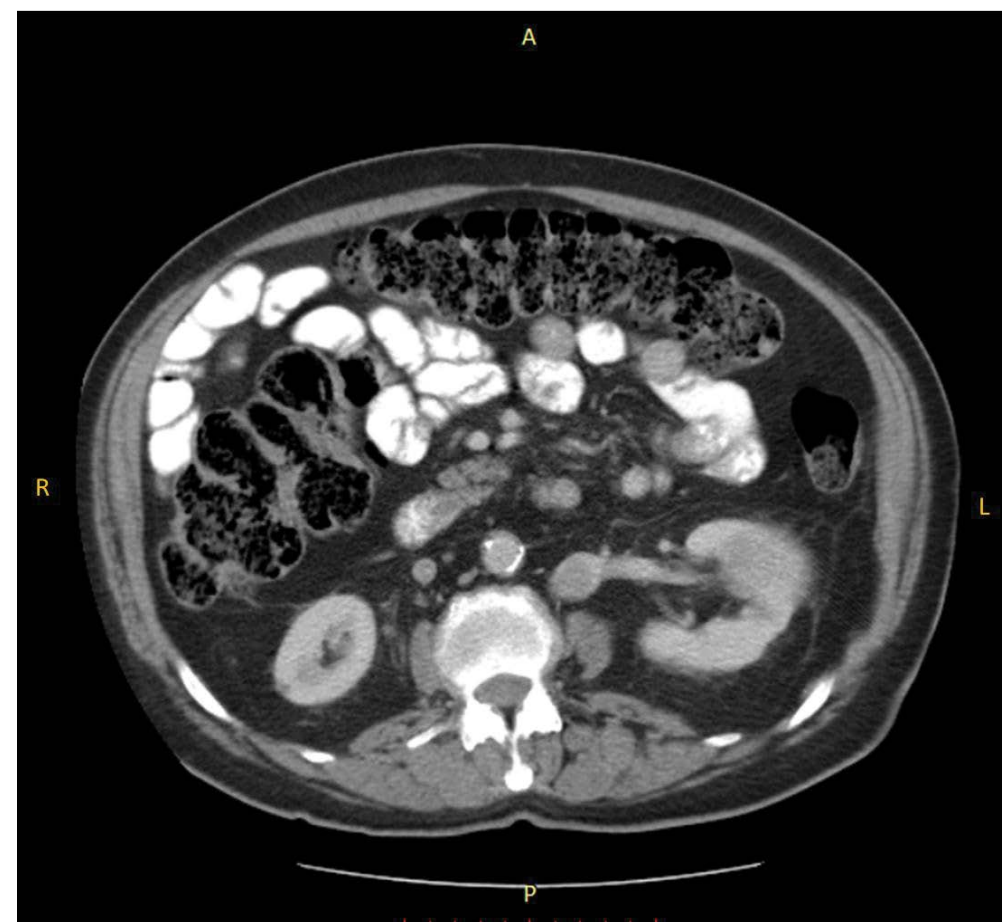

Figure 1: IVC located in the left of the midline as well as total aplasia of superior vena cava.

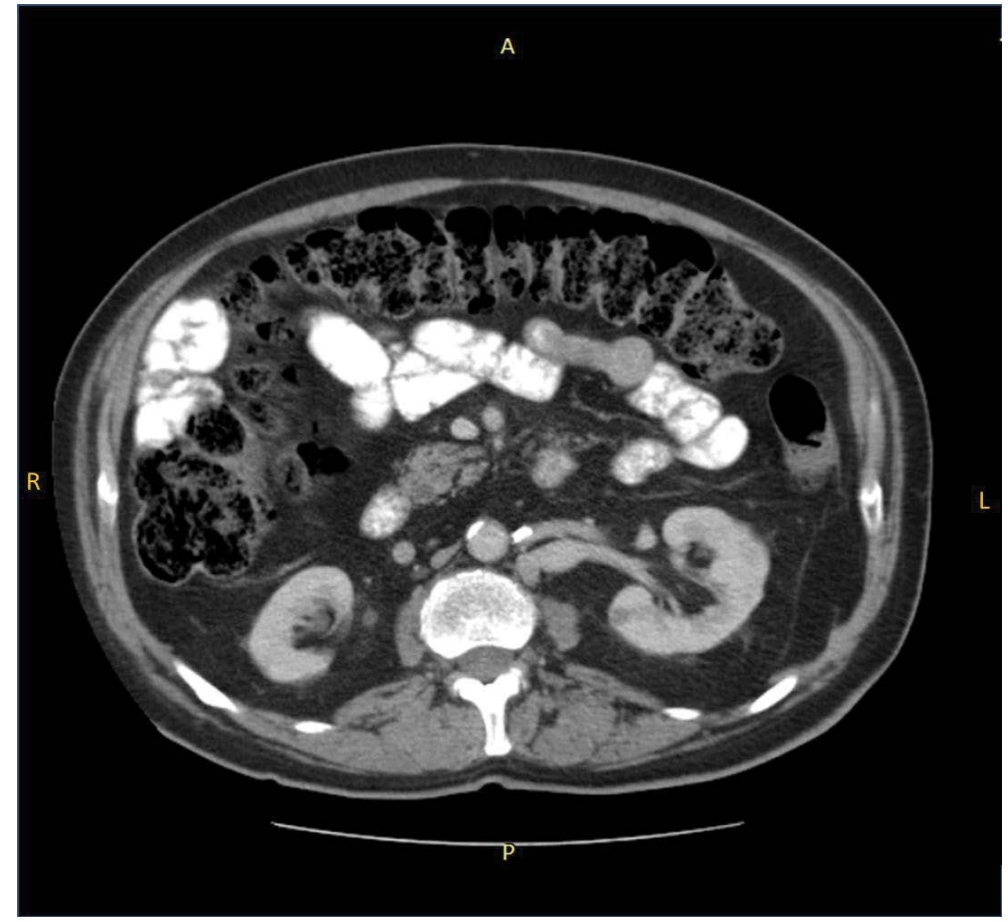

Figure 2: Left renal vein was drained straight to the IVC but as IVC was located to the left side, it did not pass in front of the aorta as usual.

the General Hospital of Athens, "G. Gennimatas", located in Athens, Greece. The study complied with the principles of Declaration of Helsinki for protection of human rights. All patients were informed in detail by the treating physician for inclusion in the study and signed an informed consent prior to participation.

\section{Declaration of Patient Consent}

The authors certify that they have obtained all appropriate patient consent forms. In the form the patient has given his consent for his images and other clinical information to be reported in the journal. The patient understands that his name and initials will not be published, and due efforts will be made to conceal his identity, but anonymity cannot be guaranteed.

\section{Financial Support Information}

There was no funding support for the conduction of this paper. 


\section{References}

1. Petik B (2015) Inferior vena cava anomalies and variations: Imaging and rare clinical findings. Insights Imaging 6: 631639.

2. Kaufman JA, Waltman AC, Rivitz SM, Geller SC (1995) Anatomical observations on the renal veins and inferior vena cava at magnetic resonance angiography. Cardiovasc Intervent Radiol 18: 153-157.
3. Giordano JM, Trout HH (1986) Anomalies of the inferior vena cava. J Vasc Surg 3: 924-928.

4. McAllister M, Bhayani SB, Ong A, Jaffe W, Malcowicz BS, et al. (2004) Vena caval transection during retroperitoneoscopic nephrectomy: Report of the complication and review of the literature. J Urol 172: 183-185. 